\title{
Appendix stump closure with titanium clips in laparoscopic appendectomy
}

\author{
Alexander Rickert • Roderich Bönninghoff • \\ Stefan Post • Markus Walz • Norbert Runkel • \\ Peter Kienle
}

Received: 22 June 2011 / Accepted: 24 October 2011 / Published online: 12 November 2011

(C) The Author(s) 2011. This article is published with open access at Springerlink.com

\begin{abstract}
Purpose Different techniques, including clips, have been used to close the stump in laparoscopic appendectomy. The aim was to investigate the results after application of a newly developed titanium clip for this operation.

Methods From June 2008 to February 2010, 104 patients from two different hospitals undergoing laparoscopic appendectomy were included in this prospective study. Closure of the appendix base was generally intended with a titanium double-shanked clip (DS-Clip). The variables of interest were intra-and postoperative complications, operation time and hospital stay. Furthermore, an evaluation of the clip's practicability by the surgeon was performed using a standardised questionnaire.

Results In 104 patients screened intraoperatively, four patients had to be excluded as the operating surgeon felt that the clip was not adequate for closing the stump, generally because of severe inflammation of the base of the appendix with involvement of the caecum. One patient developed an intra-abdominal abscess which had to be drained interventionally; no reoperations were necessary. The overall complication rate, the operation time and the hospital stay were well comparable with other devices for appendix stump closure such as staplers, loops or polymeric
\end{abstract}

\footnotetext{
A. Rickert $\cdot$ R. Bönninghoff $\cdot$ S. Post $\cdot$ P. Kienle $(\bowtie)$

Department of Surgery, University Hospital Mannheim,

University of Heidelberg,

Theodor-Kutzer-Ufer 1-3,

68167 Mannheim, Germany

e-mail: peter.kienle@umm.de

M. Walz $\cdot$ N. Runkel

Department of General Surgery, Schwarzwald-Baar-Klinikum,

Teaching Hospital University of Freiburg,

78050 Villingen-Schwenningen, Germany
}

clips. The practicability of the clip was mainly rated as excellent or good by the operating surgeons.

Conclusion This study suggests that the presented titanium DS-Clip is a safe and cost-effective technique for securing the appendix base in laparoscopic appendectomy. The application is easy and can be learned quickly, making it a good option also for teaching hospitals.

Keywords Laparoscopic appendectomy · Appendix stump closure $\cdot$ Acute appendicitis $\cdot$ Clip closure

\section{Introduction}

Appendectomy for acute appendicitis is the most common emergency surgical procedure, with more than 130,000 operations per year in Germany. More than half of these operations are nowadays performed laparoscopically [1]. Several studies and a systematic review have demonstrated at least equivalence for laparoscopic and open appendectomy $[2,3]$. Less postoperative pain, reduced wound infections, faster recovery and shorter hospital stay have been shown to be advantageous in laparoscopic compared with open appendectomy. Disadvantages of the laparoscopic operation are, besides longer procedure times, a marginally higher intra-abdominal abscess rate and higher costs [4-6]. Both latter factors are inevitably influenced by the technique of stump closure. Different ways of closing the appendix stump in laparoscopic appendectomy have been described [7, 8]. Mainly two techniques are used: first, using the Roeder loop, a pre-tied sliding knot, which is applied to both sides of the dissection line of the appendix [9, 10]; second, dissection and closure with a linear stapler (Endo GIA) [7]. Some data suggest that the stapling technique results in the safest closure of the stump, but it is also the most expensive 
method [7]. Recent studies have described the use of nonabsorbable plastic clips [11, 12]. Advantages of these clips were easy application and low costs. Due to their size, these clips are limited to an appendix base smaller than $10 \mathrm{~mm}$. In the presented pilot study, we used a titanium double-shanked clip (DS-Clip). The aim was to investigate the safety and feasibility of the clip as an alternative for stump closure.

\section{Materials and methods}

Two hospitals included patients into this prospective study: the county hospital Schwarzwald-Baar-Klinikum and the University Hospital Mannheim. The projected aim was to include 50 patients per hospital for the outcome analysis. From June 2008 to February 2010, 104 patients undergoing laparoscopic appendectomy in the two participating hospitals were screened for the study. The surgeons were instructed to always use the clip; only in cases where they intraoperatively felt that the technique was not suitable for adequate closure of the stump they could use another device (i.e. in case of a very broad appendix base or in a severely inflamed appendix with involvement of the caecum). All operations were done by a board certified surgeon or by a surgical trainee (first to sixth year of surgical training) under supervision of the former.

The data collected included age, gender, operation time, complications, management of complications, hospital stay, (histological) type of appendicitis and diameter of appendix base. The histological findings were divided into five groups: (1) no signs of acute inflammation, (2) phlegmonous appendicitis, (3) ulcerophlegmonous appendicitis, (4), gangrenous appendicitis and (5) chronic appendicitis. Data were recorded prospectively and entered into a database.

Every surgeon had to fill in a standardised form describing the intraoperative situation and the clip application. The features of the clip were rated by answering the following questions by grading them from " 1 " for excellent to "4" for poor:

1. How do you judge the fitting of the clip?

2. How do you judge the view on the applicator and the clip?

3. How do you judge the handling of the clip and the applicator?

4. Overall rating of the clip

The operation followed a standardised course. The patients were placed in supine position with the surgeon and assistant standing on the left side and the monitor on the right. The camera trocar was introduced in an open technique according to Hasson [13]. After establishing a pneumoperitoneum, a 5-mm trocar was introduced in the right lower abdomen and a $12.5-\mathrm{mm}$ trocar was introduced in the left lower abdomen under vision. After exploration of the whole abdominal cavity and confirmation of the diagnosis, the appendix was mobilised by dissecting the mesoappendix with electric coagulation. Only in cases with a very large artery a clip was applied. A clip was then placed on the base of the appendix. Another clip was placed on the distal part (Fig. 1). After cutting between the clips, the appendix was removed through the $12.5-\mathrm{mm}$ trocar.

The titanium double-shanked clip (DS-Clip) was provided by Aesculap AG (Tuttlingen, Germany). It is a pure titanium clip with two parallel shanks which are connected in the tip area of the clip (Fig. 2). The clip has to be applied with a reusable specific clip applicator and the closing mechanism initiates at the tip of the clip, which should reduce slipping off from any grasped tissue.

Statistical analyses were performed using SPSS for Windows 15.0 (SPSS Inc., Chicago, IL, USA). Fisher's exact test was used for qualitative and the $t$ test for normally distributed data.

\section{Results}

Of the 104 patients screened for this prospective observational study, 100 patients were finally included in the study (59 females, 41 males). In four cases the clip was not used because the surgeon thought it was not suitable due to severe inflammation of the appendix bases with involvement of the caecum; in these patients either a stapler (Endo GIA) or a Roeder loop was used.

The median age was 30.6 years (range, 13-84 years). The median operation time was $54 \mathrm{~min}$ (range, 26-109 $\mathrm{min}$ ). Clinical findings showed phlegmonous appendicitis in $72 \%$, gangrenous appendicitis in 19\% and no macroscopic signs of inflammation in $9 \%$. Of the 19 patients with gangrenous appendicitis, eight had a perforation and five a perityphlitic

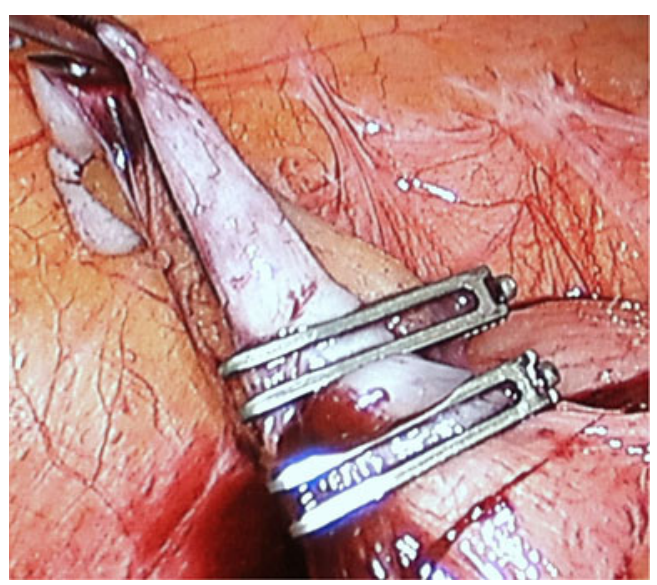

Fig. 1 Intraoperative view of applied DS-Clips in laparoscopic appendectomy 


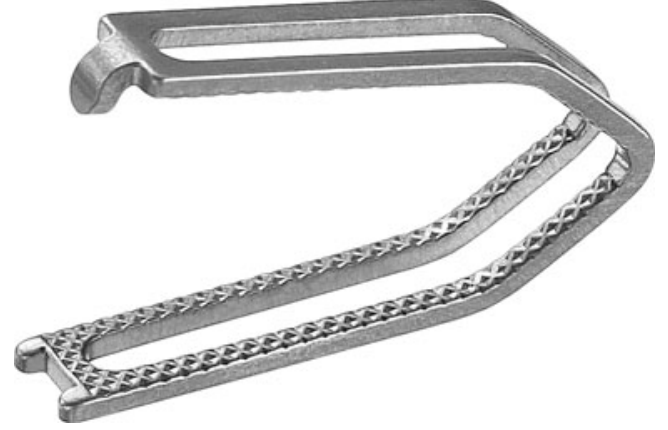

Fig. 2 Titanium double-shanked clip (DS-Clip). Picture provided by and with copyright of Aesculap AG

abscess. In case of macroscopic non-inflamed appendix and no other obvious abdominal pathology explaining right lower quadrant pain, our policy is to still remove the appendix in order to rule out other causes of pain such as neurogenic appendicitis. In this study, four patients had laparoscopy for chronic lower right abdominal pain. In one patient an enlarged, non-inflamed appendix was removed; here histology revealed a neuroendocrine tumour.

The histological findings are shown in Fig. 3. The clip was applied in 23 cases onto an inflamed appendix base. The median diameter of the appendix base was $10 \mathrm{~mm}$ (range, $5-15 \mathrm{~mm}$ ). The maximum diameter of the appendix was $15 \mathrm{~mm}$.

The rate of surgical complications requiring specific treatment was $3 \%$. One abdominal abscess was treated by interventional drainage. One patient experienced a prolonged small bowel obstruction, which resolved under conservative treatment (nasogastric tube, bowel stimulation). One wound infection occurred, which resolved under antibiotic therapy. All of these complications occurred in the 13 patients with perforated appendicitis or a perityphlitic abscess. The complication rate in this group was $20 \%$ compared to $0 \%$ in patients with uncomplicated appendicitis $(p=0.004)$.

The median postoperative hospital stay was 4 days (range 2-19 days). Overall, 22\% of the operations were

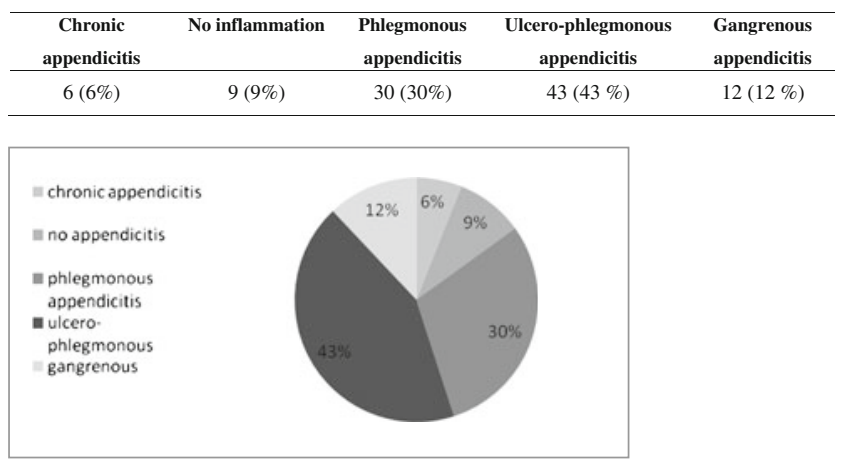

Fig. 3 Postoperative histological findings done by surgical trainees. Patient characteristics and results are shown in Table 1.

Statistically significant differences between the two hospitals were found in regard to age, number of operations done by surgical trainees $(8 \%$ in the county hospital vs. $36 \%$ in the university hospital) and procedure time (45 vs. $62 \mathrm{~min}$ in favour of the county hospital) (Table 2). The evaluation of the clip by the operating surgeons is shown in Fig. 4 with an overall judgement of the clip of $49 \%$ "excellent", $49 \%$ "good", and 2\% "average".

\section{Discussion}

Laparoscopic compared to open appendectomy has been shown to be advantageous in regard to early postoperative parameters such as postoperative pain and recovery of bowel function and also harbours a lower wound infection rate. But there is concern that there may be a higher rate of intra-abdominal abscesses, especially in perforated appendicitis [14]. Possible reasons for this observed trend in the studies are dissemination of infectious material throughout the abdomen during pneumoperitoneum and the noninversion of the appendix stump as generally done in open appendectomy. The technique of stump closure may play an important role in this context [4].

Most surgeons use either a linear stapler (endo GIA) or an endo loop to secure the appendix base. Two systematic reviews have compared these two techniques $[7,8]$. Both

Table 1 Characteristics and outcome of all patients undergoing laparoscopic appendectomy and stump closure with a titanium double-shanked clip (DS-Clip)

\begin{tabular}{lcl}
\hline & $N=100$ & \\
\hline Age (years) & 30.6 & (range 13-87) \\
Gender female & 59 & \\
Intraoperative findings & & \\
Phlegmonous appendicitis & 72 & \\
Gangrenous appendicitis & 19 & \\
$\quad$ - perforated appendicitis & 8 & \\
$\quad$ - perityphlitic abscess & 5 & \\
No appendicitis & 9 & \\
Intraoperative complications & 0 & \\
Operation time (min) & 54 & (range 26-109) \\
Operation done by surgical trainee & 22 & \\
Hospital stay (days) & 4 & (range 2-19) \\
Mean appendix diameter (mm) & 10 & (range 5-15) \\
Postoperative complications & & \\
Abdominal abscess & 1 & \\
Postoperative ileus & 1 & \\
Wound infection & 1 & \\
\hline
\end{tabular}


Table 2 Characteristics and outcome of patients undergoing laparoscopic appendectomy and stump closure with a titanium double-shanked clip (DS-Clip) stratified according to hospital

\begin{tabular}{lccl}
\hline & County hospital & University hospital & $P$ value \\
\hline$N$ & 48 & 52 & \\
Age (years) & 32.5 (range 13-82) & 28.1 (range 15-87) & $<0.0001$ \\
Gender female $(n(\%))$ & $22(44 \%)$ & $37(71 \%)$ & n.s. \\
Operation time (min) & $45($ range 26-90) & $62($ range 31-109) & $<0.0001$ \\
Operation done by surgical trainee & $4(8 \%)$ & $18(36 \%)$ & $<0.01$ \\
Hospital stay (days) & 4 (range 3-14) & 3 (range 2-19) & n.s. \\
\hline
\end{tabular}

manuscripts revealed shorter procedure times, lower rates of wound infection and postoperative ileus in favour of the stapler group; overall perioperative complication rates and hospital stay were comparable. The endo-loop technique was significantly less costly. Although both reviews yielded similar results, different conclusions were drawn. While Kazemier et al. generally recommend the use of staplers, Sajid et al. concludes that Endo loops may safely be used and are preferable for securing the appendix stump [7, 8].

Recently, the application of clips as an alternative option to close the appendix base in laparoscopic appendectomy has been described. Clips are quite commonly used in laparoscopic surgery, i.e. for the ligation of vessels or the cystic duct. Several papers describe the use of polymeric non-absorbable clips (Hem-o-lok) as a safe, feasible and cost-effective method $[11,12,15]$. In these studies one or two clips are placed onto the appendix stump. The largest, recommended diameter of the appendicular stump that can be safely closed with this clip is $10 \mathrm{~mm}$. However, the base of the appendix may often exceed this diameter in acute inflammation. In our study we used a titanium doubleshanked clip (DS-Clip). One clip was applied to the appendix base and another to the distal part which was removed. Appendix stumps with a diameter of up to $20 \mathrm{~mm}$ could be safely closed. Due to the design of the clip as a double-shanked clip, application of only one clip on each side was generally sufficient. An obvious advantage of the titanium clip in comparison to other commercially available clips is the size which allows closure of an appendix base larger than $10 \mathrm{~mm}$. Moreover, due to its closing mechanism, which first closes at the distal end and then gradually approximates between both ends, the phenomenon of "pushing out" of the tissue, in this case the appendix, can be diminished. In $23 \%$ of the cases, the inflammation involving the appendix stump and the clip could still be used without major problems. The size of the applicator requires a 12.5-mm trocar, which is equivalent to the trocar size needed when using a stapler. However, in comparison to the other commercially
Fig. 4 Evaluation of the titanium double-shanked clip (DS-Clip) by the operating surgeon. The evaluation was performed in regard to the following subjective items: fitting of the clip, view on the applicator and clip, handling of the applicator and the clip and overall rating of the clip. The following four grades were used: $1=$ "excellent", $2=$ "good", $3=$ ="average", $4=$ ="poor"

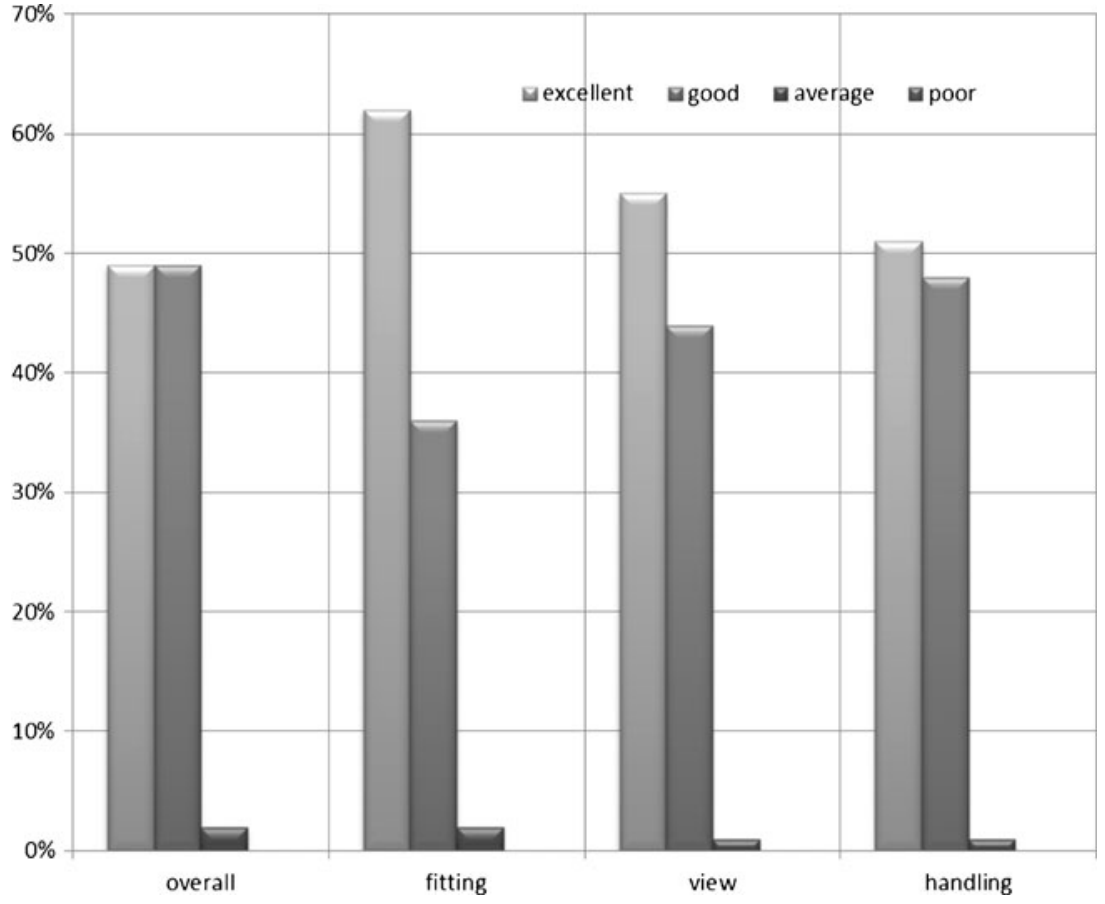


available clips, which go through a $10-\mathrm{mm}$ trocar, this is a certain drawback. The fascias in $12.5-\mathrm{mm}$ trocar incisions generally need to be closed in order to prevent trocar hernias; $10-\mathrm{mm}$ trocar incisions are generally not closed.

The objective parameters in our study such as complication rate, procedure time and length of hospital stay were well comparable to other studies using staplers, endo loops or polymeric non-absorbable clips for appendix stump closure $[2,12,15]$. None of the complications in the presented study required re-operation.

As complications only occurred in patients with perforated appendicitis or a perityphlitic abscess, the question arises whether the clip is also safe in these patients. The single patient with a postoperative intra-abdominal abscess was treated by interventional drainage. Therefore it remains unclear whether the abscess was indeed caused by leakage of the appendiceal stump or was the result of ongoing local peritonitis. But even if the former was the case, the overall complication rate in the sub-group of perforated appendicitis in our series is well comparable to the complication rate in other studies [11, 15]. Therefore these complications are probably not a result of the clip per se, but merely reflect the more difficult situation in complicated appendicitis.

Besides these objective variables, the subjective rating of the surgeons which used the DS-Clip was positive in regard to handling, visualization and fitting of the clip. The study also shows that the clip is well applicable in the setting of a teaching hospital with a high percentage of operations being done by surgical trainees.

Finally, compared to the use of linear staplers, the application of two to three of such clips will significantly reduce the costs. The pricing for a set of four clips is predicted to be around $80 €$ depending on the region. Further studies with a larger patient number will have to confirm the safety of the new clip and also investigate the possible cost advantage.

\section{Conclusion}

This study suggests that the presented titanium doubleshanked clip is a safe and cost-effective technique for securing the appendix base in laparoscopic appendectomy. An appendix base with a diameter of up to $20 \mathrm{~mm}$ can be adequately closed, which is a possible advantage in comparison to other commercially available clips. The application is easy and can be learned quickly, making it a good option also for teaching hospitals.
Acknowledgements S.P., N.R. and P.K. received payments for lectures and courses for Aesculap AG.

Conflicts of interest None.

Open Access This article is distributed under the terms of the Creative Commons Attribution Noncommercial License which permits any noncommercial use, distribution, and reproduction in any medium, provided the original author(s) and source are credited.

\section{References}

1. Eypasch E, Sauerland S, Lefering R, Neugebauer EA (2002) Laparoscopic versus open appendectomy: between evidence and common sense. Dig Surg 19:518-522

2. Sauerland S, Jaschinski T, Neugebauer EA (2010) Laparoscopic versus open surgery for suspected appendicitis. Cochrane Database Syst Rev CD001546

3. Gorenoi V, Dintsios CM, Schonermark MP, Hagen A (2007) Laparoscopic vs. open appendectomy: systematic review of medical efficacy and health economic analysis. GMS Health Technol Assess 2:1-12

4. Krisher SL, Browne A, Dibbins A, Tkacz N, Curci M (2001) Intra-abdominal abscess after laparoscopic appendectomy for perforated appendicitis. Arch Surg 136:438-441

5. Sporn E, Petroski GF, Mancini GJ, Astudillo JA, Miedema BW, Thaler K (2009) Laparoscopic appendectomy - is it worth the cost? Trend analysis in the US from 2000 to 2005. J Am Coll Surg 208:179-185

6. Kockerling F, Schug-Pass C, Grund S (2009) Laparoscopic appendectomy. The new standard? Chirurg 80:594-601

7. Kazemier G, in't Hof KH, Saad S, Bonjer HJ, Sauerland S (2006) Securing the appendiceal stump in laparoscopic appendectomy: evidence for routine stapling? Surg Endosc 20:1473-1476

8. Sajid MS, Rimple J, Cheek E, Baig MK (2009) Use of endo-GIA versus endo-loop for securing the appendicular stump in laparoscopic appendicectomy: a systematic review. Surg Laparosc Endosc Percutan Tech 19:11-15

9. Semm K (1983) Endoscopic appendectomy. Endoscopy 15:59-64

10. Arcovedo R, Barrera H, Reyes HS (2007) Securing the appendiceal stump with the Gea extracorporeal sliding knot during laparoscopic appendectomy is safe and economical. Surg Endosc 21:1764-1767

11. Partecke LI, Kessler W, von BW, Diedrich S, Heidecke CD, Patrzyk M (2010) Laparoscopic appendectomy using a single polymeric clip to close the appendicular stump. Langenbecks Arch Surg 395:1077-1082

12. Delibegovic S, Matovic E (2009) Hem-o-lok plastic clips in securing of the base of the appendix during laparoscopic appendectomy. Surg Endosc 23:2851-2854

13. Hasson HM (1984) Open laparoscopy. Biomed Bull 5:1-6

14. Guller U, Hervey S, Purves H, Muhlbaier LH, Peterson ED, Eubanks S, Pietrobon R (2004) Laparoscopic versus open appendectomy: outcomes comparison based on a large administrative database. Ann Surg 239:43-52

15. Hanssen A, Plotnikov S, Dubois R (2007) Laparoscopic appendectomy using a polymeric clip to close the appendicular stump. JSLS 11:59-62 